\title{
Disc Herniation and Knee Arthritis as Chronic Oxidative Stress Diseases: The Therapeutic Role of Oxygen Ozone Therapy
}

Emma Borrelli ${ }^{1}$, Alberto Alexandre $^{2}$, Emmanouil Iliakis $^{3}$, Andrea Alexandre $^{4}$ and Velio Bocci ${ }^{5}$

${ }^{1}$ Department of Medical Biotechnologies, University of Siena, Siena, Italy

${ }^{2}$ European Neurosurgical Institute, Treviso, Italy

${ }^{3}$ Orthopedic Surgeon, Hospital Inspector, Athens, Greece

${ }^{4}$ Department of Radiology, Catholic University, Rome, Italy

${ }^{5}$ Department of Chemistry, Pharmacy and Biotechnologies, University of Siena, Siena, Italy

\begin{abstract}
In this review the Authors will explain the pathophysiological mechanism of action of the oxygen ozone therapy in the treatment of the disc herniation and knee arthritis. The ozone injected in the intervertebral disc or in the joint causes a rapid oxidative reaction with the organic compound of disc and synovial fluid and a late response due the formation of various second messengers that are able to stimulate the endogenous production of antioxidants through a hormetic production of hydrogen peroxide. As a consequence of the intradiscal or intrarticular injection of ozone we noted a dramatic decrease of the pain and a reduction of the local and systemic inflammation. The ozone therapy is cost-effective and should be used to improve recovery of the disc herniation and knee arthritis.
\end{abstract}

Keywords: Disc herniation; Knee arthritis; Oxygen ozone therapy; Chronic oxidative stress; Hormesis

\section{Introduction}

Despite its large and worldwide diffusion among physicians, oxygen-ozone therapy seems more or less deliberately ignored by the medical academic community. This is probably because often the ozone therapy has been used improperly or because most of the ozone therapists were interested in the rapid application of this therapy on patients instead of seeking the mechanism of action of ozone in the human body. In the last decade, a large number of biochemical studies on the ozone therapy were published allowing to clarify why the ozone can be considered really a drug.

The aim of this review is to present the state of the art of the ozone therapy in the treatment of two important chronic oxidative stress diseases, the disc herniation and the knee arthritis, starting from the intracellular effects of the ozone up to its efficacy in symptoms reduction.

\section{Disc herniation and knee arthritis as chronic oxidative stress diseases}

Normal aerobic metabolism is associated with unavoidable production of free radicals and related reactive oxygen/nitrogen species (ROS and RNS). At low to moderate concentrations, they function in physiological cell processes, but at high concentrations they produce adverse modifications to cell components such as lipids, proteins and DNA. Aerobic organisms have integrated antioxidant systems which include enzymatic and non-enzymatic antioxidants that are usually effective in blocking harmful effects of ROS. However in pathological conditions the antioxidant systems can be overwhelmed. The shift in balance between oxidant/antioxidant in favor of the oxidant was defined by Sies as oxidative stress [1-3].

A large number of studies reported the central role of the oxidative stress in the development of the chronic degenerative diseases like cancer, cardiovascular diseases, and diabetes and is considered an important factor in aging [4]. Moreover, there are emerging evidences indicating that oxidative stress is also involved in progressive degenerative diseases of different skeletal tissues.
For example in disc tissues, oxidative stress may initiate or participate in matrix destruction and cell apoptosis, leading to a disc degeneration. In cultured disc cells, the increase of nitric oxide suppresses proteoglycan synthesis and the addition of hydrogen peroxide causes apoptosis of the human cells of annulus fibrous. Brown degeneration of the collagen framework is thought to be closely associated with age-related disc degeneration and is a consequence of the non-enzymatic glycoxidation of matrix lysyl residues under cumulative oxidative stress [5-7].

As far as the joint disease, proinflammatory factors such as cytokines and prostaglandins are released at sites of inflammation, together with ROS and NO. These factors are associated with very low Superoxide Dismutase concentrations in joint fluids and an increase of ROS. In the articular cartilage advanced glycation end products (AGEs) was found and there is a correlation between increased osteoarthritis severity and cartilage AGE levels $[8,9]$.

On the basis of these assumptions, the first therapeutic approach in chronic oxidative stress diseases was the oral supplementation of synthetic antioxidants; surprisingly, the antioxidant administration can be deleterious for the organism and can lead to cancerogenesis and aging [10]. The term of "antioxidative stress" is related to an excessive increase in antioxidants and it has been reported that antioxidants, if oversupplied, abolish the life extension by inhibiting a process called mitohormesis. It appears that the complete elimination of free radicals would thus disrupt, rather than extend, the normal functioning of the body [11].

*Corresponding author: Emma Borrelli, Department of Medical Biotechnologies Policlinico Le Scotte, Viale Bracci 1, 53100 Siena, Italy, Tel: 00393282679264 E-mail: ebitaly2007@libero.it

Received July 07, 2015; Accepted August 08, 2015; Published August 15, 2015 Citation: Borrelli E, Alexandre A, lliakis E, Alexandre A, Bocci V (2015) Disc Herniation and Knee Arthritis as Chronic Oxidative Stress Diseases: The Therapeutic Role of Oxygen Ozone Therapy. J Arthritis 4: 161. doi:10.4172/2167 7921.1000161

Copyright: $\odot 2015$ Borrelli E, et al. This is an open-access article distributed under the terms of the Creative Commons Attribution License, which permits unrestricted use, distribution, and reproduction in any medium, provided the original author and source are credited. 
The beneficial physiological cellular use of ROS is now being demonstrated in different field, including intracellular signals and redox regulation. It has been documented that low levels of ROS are signaling molecules, modulating cell proliferation and apoptosis, gene expression through activation of transcription factors like nuclear factor kappa beta (Nfk $\beta$ ) and hypoxia inducible factor (HIF). Paradoxically the best method of defense and repair during chronic oxidative stress may be oxidative stress itself with controlled exposure to ROS, since the expression of many DNA repair enzymes is upregulated during the oxidative stress. The terms "hormesis" describes beneficial effects after an exposure to a low-intensity stressor. Studies reported the life prolonging effects of aldehydes, hydrogen peroxide $\mathrm{H}_{2} \mathrm{O}_{2}$, physical exercise, caloric restriction [12]. Whether ROS will act as damaging, protective or signaling factors depends on the delicate equilibrium between ROS production and scavenging at the proper time and side. On this pathophysiological basis, the use of the ozone in the treatment of the chronic oxidative stress diseases appears promising. Biochemical and clinical studies suggested that ozone, when contact biological fluids of the body, is able to produce a small amount of hydrogen peroxides and stimulate the production of endogenous antioxidants [13]. Clinical studies reported the efficacy of the major ozonated autohaemotherapy (MOAT) on chronic oxidative stress diseases like chronic obstructive pulmonary disease (COPD) age related macular degeneration (ARMD), and chronic ulcers [14-16].

In the next chapters we will try to explain how the ozone acts in the treatments of the disc herniation and the knee arthritis when used as systemic therapy (MOAT) or as local injection (intradiscal or intra articular injection)

\section{The ozone}

Ozone is the third strongest oxidant after fluorine and persulphate and in presence of organic compounds immediately reacts and generates a large number of oxidized molecules, disappearing in few seconds. Ozone is an air pollutant and it is undoubtedly toxic when inhaled. The reaction between ozone and the epithelial lining fluid of the lung causes an acute inflammatory generalized reaction [17]. If the ozone is used as therapeutic drug, the ozonation process either happening in blood, or intradiscal or in an intrarticular site represents an acute oxidative stress. However, provided that it is precisely calculated according to a judicious ozone dosage, it is not deleterious but it is actually capable of eliciting a multitude of useful biological responses and, possibly, reversing a chronic oxidative stress due to ageing, chronic infections, diabetes, atherosclerosis, degenerative processes and cancer. Indeed the ozono therapeutic act is interpreted as an atoxic but real "therapeutic shock" able to restore homeostasis [18]. In the first years of the $19^{\text {th }}$ century some physicians start to use the ozone in the treatment of cutaneous gangrene of German soldiers. Due the potent antibacterial capacity, the ozone was effective in the local disinfection of wounds and fistulae. In the middle of age 90, Hans Wolff first developed the method of autohaemotherapy by insufflating the ozone into the blood contained in a disposable ozone-resistant gas bottle. In the same years, the techniques of intrarticular and intradiscal ozone injection were performed.

After the explanation of the mechanism of action of the ozone in biological fluids, clinical studies on the efficacy of ozone when injected in the disc, in paravertebral muscles or in the joints were published and at this moment is well known the pain and inflammation reduction after intradiscal and intrarticular ozone injections in patients with disc degeneration or osteoarthritis.

\section{The biochemical mechanism of action of ozone}

A complete description of the mechanism of action of ozone was reported in previous works $[19,20]$. Briefly, ozone dissolves physically in pure water and in biological water (physiological saline, plasma, lymph, urine) according Henry's law in relation to the temperature, pressure and ozone concentration. During the major ozonated autohaemotherapy, for example, ozone immediately reacts with plasmatic antioxidants, polyunsaturated fatty acids (PUFA), proteins and carbohydrates. Thus, ozone leads to the formation of Reactive oxygen species (ROS), lipid oxidation products (LOP) and oxidized antioxidants producing immediately two crucial messengers: hydrogen peroxide, one of the reactive oxygen species, and aldehydes such as 4-hydroxy-2-nonenal (4-HNE) as the result of PUFA peroxidation. $\mathrm{H}_{2} \mathrm{O}_{2}$ is a common compound in blood cells and, by entering into the mass of erythrocytes activates glycolysis with a transient increase of ATP and of 2-3-diphosphoglycerate (2,3-DPG). This compound causes a shift to the right of oxyhemoglobin and this very useful process improves the release of oxygen into ischemic tissues, an important step in ischemic diseases. 4-HNE produced during PUFA peroxidation circulates all over the body and particularly in chronically inflamed organs and can enter into cells undergoing chronic oxidative stress. 4-HNE, by its binding to either Cys 273 or Cys 288 of Keap 1 allows the release of the transcription factor nuclear releasing factor 2 ( Nrf2), normally inactive because bound to a large protein called Keap1(Kelch-like ECH associated protein),which then transfers into the cell nucleus and binds to the antioxidant response element (ARE) of gene promoters. This induces the expression of over 230 genes able to provide the synthesis of potent cellular antioxidant and detoxification systems.

The induced genes include the synthesis of glutathione (GSH), GSH reductase, GSH transferase, GSH peroxidases, thioredoxin, thioredoxin reductase, nicotinamide adenin dinucleotide phosphate, reduced form $(\mathrm{NADPH}), \mathrm{NAD}(\mathrm{P})$-quinone oxidoreductase 1 (NQO1), uridine diphosphate glucose (UDP) syaliltransferase and heme-oxygenase. Nrf2 also plays a role in the negative regulation of insulin and growth hormone which increases stress resistance [21]. When ozone is injected in the intervertebral disc or in the joint we observe a rapid oxidative reaction with the organic compound of disc and synovial fluid and a late response due the formation of various second messengers. The next few paragraphs will explain the reactions in details. As a consequence, the use of ozone both locally and with major ozonated autohaemotherapy should be encouraged as treatment of all chronic oxidative diseases, particularly in the disc herniation and knee arthritis.

\section{Materials and Methods}

\section{Ozone therapy and disc degeneration}

With increasing age and degeneration the most significant biochemical change to occur in disc is loss of protoeglycans. These proteoglycans enable the nucleus pulposus to retain water, thereby cushioning and absorbing the considerable loads placed on the tissue. With increasing age the nucleus pulposus become more fibrous and the annulus is forced to carry larger loads leading to tears, bulging, rupture and herniation. The loss of proteoglycans is also involved in the loss of aggrecan and type II collagen with subsequent penetration of large molecules like growth factor and cytokines in the disc. Progression of disc degeneration is probably related to molecular events mediated by nuclear factor kappa beta and mitogen-activated protein kinases (MAPKs). Pain is probably associated with a nerve growth in the annulus fibrosus, usually poorly innervated. The 
inflammation promotes the growth of afferent fibers in the disc and this growth might be the cause of discogenic low back pain [22-25]. The application of ozone in the herniated disc and low back pain has proved very effective. It can be administered directly (intradiscal) or indirectly via intramuscular administration into the paravertebral muscles. The direct intradiscal injection uses a variable volume of gas (3-10 ml) at an $\mathrm{O}_{3}$ concentration of 20-30 micrograms $/ \mathrm{ml}$. With this minimally invasive technique several thousand patients have been treated with a success rate of $70 \%$ [26]. In the case of a gas injection directly into the nucleus pulposus, ozone exerts a multiplicity of effects, such as the activation of the anti-nociceptive system, and it has anti-inflammatory action due to lipid peroxidation products, with the consequent inhibition of cyclooxygenase-2. We have some evidence that ozone dissolves in the intradiscal water and reacts with the complex macromolecular components such as proteoglycans and glicosoaminoglycans. The reaction entails an oxidation of these substrates (galactose, glycuronic acid, glycine, 4-hydroxyprolin) and the breakdown of intra- and intermolecular chains leading to a disintegration of the three- dimensional structure. Its collapse frees the entrapped water that, after reabsorption, allows a decrease of intradiscal pressure and possibly a disappearance of pain due to the reduced pressure on the nervous root. Probably ozone also destroys the nerve growth in the annulus fibrosus with a rapid reduction of the pain. However, because ozone is very often released also along the injection path (i.e., intraforaminal), the final therapeutic effect is due to the combination of a vasculomediated and biochemical effects (improved oxygenation, correction of local acidosis, disappearance of venous and lymphatic stasis). It seems important to postulate that in the intraforaminal space, the presence of lipids, an excellent substrate for ozone, may favor the release of oxidized phospholipids to be included among LOPs: surprisingly, during inflammation, these compounds can inhibit inflammation as it has been shown in mice undergoing a lethal endotoxic shock. Thus, ozone appears to display paradoxical and unexpected useful effects such as inhibition of the release or inactivation of proteinases and likely enhancement of the release of immunosuppressive cytokines such as TGFß or/and IL-10. Even more surprising is the recent evidence suggesting that ozone, by inhibiting COX-2, blocks the synthesis of proinflammatory prostaglandin $\mathrm{E}_{2}$. Another important analgesic effect may be derived by the induction of antioxidant enzymes resulting in the adaptation to chronic oxidative stress. These novel and surprising aspects have allowed formulating the concept of the ozone-paradox $[27,28]$.

Unfortunately there are few experimental studies on the technical aspect of the intradiscal injection. As reported above, there is a large variability on pressure of injection, volume of gas injected, concentration of ozone used in therapy. To evaluate if disc herniation can produce not only a local free radical increase but also a systemic oxidative stress disease we recently performed a pilot clinical study on 40 male and homogeneous patients with disc herniation (unpublished data). All patients between 18 and 70 years of age with sciatica since 6-8 weeks are eligible for inclusion in the study. A disc herniation at the appropriate level will have to be shown by Magnetic Resonance Imaging. The herniated fragment has to be smaller than $1 / 3$ of the spinal canal. Patients were enrolled in the study from February 2014 to February 2015. all subjects received one intradiscal injection of oxygen-ozone (following the method reported in Buric et al. [28]) and eight major ozonated autohaemotherapy two times a week $(150 \mathrm{ml}$ of blood $+150 \mathrm{ml}$ of ozone at a concentration of $20 \mathrm{micrograms} / \mathrm{ml}$ of gas according to Borrelli et al.) [14]. Before and after the last treatment of MOAT we analyzed in all patients the plasma value of Reactive Oxygen
Metabolites (d-ROM test, Diacron International, Grosseto, Italy) and the SH proteins (Diacron International, Grosseto, Italy) to better understand the time course of the oxidative stress. Before the use of ozone therapy we found in patients a marked increase in ROM value and a decrease in protein $\mathrm{SH}$ content in plasma. After the associated treatments intradiscal ozone + MOAT a slight increase of protein $\mathrm{SH}$ and a decrease in ROM (reduction in plasma oxidative stress index) were observed. These preliminary results suggest a beneficial action of the combined treatments (intradiscal plus MOAT) in the reduction of low back pain and disability following disc herniation.

\section{Ozone therapy and knee arthritis}

Usually a fibrous joint capsule surrounds the ends of the bones in the joint and contains the synovial fluid-filled cavity. This organ is comprised of specialized tissues that enable a frictionless environment essential for movement and transfer of load from one bone to another. Articular cartilage is mostly composed of water and proteins of extracellular matrix, with a sparsely distributed population of chondrocytes. The major matrix proteins in cartilage are the proteoglycans (aggrecan) and collagen. Osteoarthritis is the most common form of arthritis that is characterized by loss of joint form and function due to progressive articular cartilage degeneration. Structural changes of the osteoarthritis of knee are visible on standards $\mathrm{X}$-ray and include narrowing of the joint space due to cartilage loss, formation of osteophytes at the joint margins and bony sclerosis or increased density or thickness of the bone just underneath the articular cartilage [29]. As previously discussed, there are growing evidences for an involvement of the oxidative damage in the pathophysiological changes of the knee osteoarthritis. ROS are produced by chondrocytes in response to stimulation by numerous cytokines and growth factors, including Interleukin -1 (IL-1) Tumor Necrosis Factor alpha (TNF alpha) and Transforming Growth Factor beta (TGF beta). IL-1 dependent production of ROS is implicated in causing DNA damage in chondrocytes. Compared to young rats, old rats have increased levels of intracellular ROS in their cartilage. Recently the dysfunction of chondrocyte mitochondria was implicated in the establishment and progression of osteoarthritis [30,31].

In the last decades a number of orthopedics in Europe have begun to treat acute and chronic knee arthritis with intra articular or peri articular insufflations of small volume of ozone (5-10 ml with ozone concentration from 5 to 15 micrograms/ml of gas) with a rapid pain relief, decongestion, disappearance of edema, and increased mobility. Ozone, when injected in the knee, dissolves in the synovial fluid that contains antioxidants like SOD, GSH, vitamin E, PUFA and proteins and generates ROS and LOP which in turn are responsible for:

1) A possible inactivation and inhibition of the release of proteolytic enzymes and proinflammatory cytokines; 2) An induction of the proliferation of chondrocytes and fibroblasts; 3) A release of the synthesis of antioxidant enzymes (SOD,GSH-Px and catalase) as adaptive response of chronic oxidative stress; 4) A release of immunosuppressive cytokines such as TGF betal and Interleukin 10 (IL-10) that modulate the synthesis of integrins and stimulate the synthesis of matrix protein such as collagen and proteoglycans $[32,33]$.

Unfortunately there are no experimental studies on the mechanism of action of ozone in the knee arthritis, and there is a large variability in terms of side of injection, volume and concentration of gas. To partially overcome this deficiency, one of us (EI) performed two experimental studies on animals (unpublished data). In the first study, a safety of the intrarticular injection of ozone was studied on 36 male rats. Animals 
Citation: Borrelli E, Alexandre A, lliakis E, Alexandre A, Bocci V (2015) Disc Herniation and Knee Arthritis as Chronic Oxidative Stress Diseases: The Therapeutic Role of Oxygen Ozone Therapy. J Arthritis 4: 161. doi:10.4172/2167-7921.1000161

were divided in two groups: group 1 (18 rats) received a single intra articular injection of $0.05 \mathrm{ml}$ of monoiodiacetate (MIA), a well-known inflammatory agent, in the right knee, group 2 (18 rats) received in the same articulation $0.05 \mathrm{ml}$ of ozone at dose of $20 \mathrm{micrograms} / \mathrm{ml}$ three times a week for a total of 12 injections. All animals were killed at the same time after 4 weeks and histological examination was performed. In the group 1 there are a marked inflammatory response MIA related, whereas no alterations was found in group 2 rats treated with repeated dose of ozone (Figures 1 and 2).

These data suggest that ozone causes no damage in joints even if injected repeatedly. In the second experimental study 24 guinea pigs were used. In all animals, arthritis was induced with intracapsular injection into the knees joint of 50 microliters of sodium monoiodacetate (MIA) through the patellar ligament. Animals were randomized in three groups:

Groups 1 ( 8 animals): 8 days after MIA administration, received 50 microliters of a gas mixture $\mathrm{O}_{2}-\mathrm{O}_{3}$ at $30 \mathrm{micrograms} / \mathrm{ml}$ in the right knee 2 times a week for five weeks. At the same time left knee was injected with oxygen $(50 \mu \mathrm{l})$ alone and served as control. Group 2 (8 animals): 11 days after MIA injection received the $\mathrm{O}_{2} \mathrm{O}_{3}$ mixture in the right knee and the oxygen in the left knee with the same procedure of group 1. Group 3 (8 animals): after 15 days of MIA injection received the ozone injection like the other groups. One week after the last oxygen ozone injection, all animals of the three groups were deeply anesthetized and sacrificed. The knee joints were the dissected to expose the articular cartilage. The cartilage was carefully dissected away from the subcondral bone using a dissecting microscope. Cartilage from the tibial plateau, medial and lateral was analysed. The histological appearance of the knee joints was evaluated by one blinded independent investigator using a modified Mankin scoring system.

In the Group 1 the knee treated with $\mathrm{O}_{2}-\mathrm{O}_{3}$ it showed an excellent architectural preservation of cartilage without sign of degeneration (Figure 3). In Group 2 we observed only a reduced degeneration in the $\mathrm{O}_{3}$ treated knee, but the process of cartilage destruction was present. In Group 3 the histology showed a marked inflammatory degeneration of the cartilage (Figure 4).

It seems that the intra -articular injection of ozone should be performed in the early stage of the osteoarthritis because ozone cannot preserve or restore the advanced inflammatory alteration of the cartilage.

\section{Discussion}

The most common age-associated conditions of humans are chronic pain and disability due to disk herniation and osteoarthritis, especially in the developed countries where the aging population is growing. Aging was traditionally assumed to cause the development of joint and disk degenerations through sequential events of mechanical wear and tear affecting the matrix [34,35]. However, a number of studies suggest a mechanism in which oxidative damage-induced cellular senescence is the primary driver of this degeneration. Senescent cells have an impaired capacity for matrix repair and synthesis and thus tissue redox homeostasis is lost. If we assumed the pivotal role of the chronic oxidative stress in the development of disk herniation and knee arthritis, the key to the future success of regulating oxidative stress induced damage should be the suppression of oxidative damage without disruption of the well-integrated antioxidant defence network. The basic concept of hormesis is to elicit the intrinsic capability of a body rather than to supply exogenous natural or synthetic antioxidants.
Ozone has an excellent hormetic property and it is possible to integrate the systemic and local administration of ozone to treat disc herniation and knee arthrosis and reduce the simptomatology. Moreover, disc herniation and knee arthrosis are two chronic oxidative stress diseases that often could require a surgical intervention. Surgery can produce satisfactory results in a high proportion of patients in the short to medium term. However, long term studies have reported variable benefits with a high proportion of patients describing persistent pain. In addition, lumbar discectomy and knee prothesis are expensive and invasive surgical procedures [36,37]. Intradiscal ozone injection is a low cost, minimally invasive procedure with direct and indirect

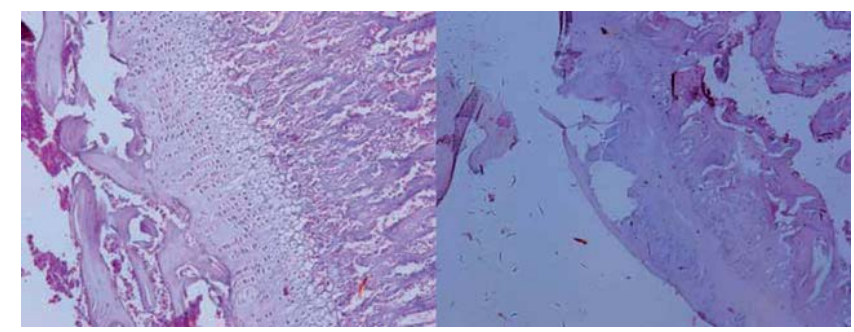

Figure 1: Total destruction of the articular cartilage of the male rat afte the infusion of sodium monoiodacetate (From Dr. Emmanouil Iliakis, 2010, personal files).

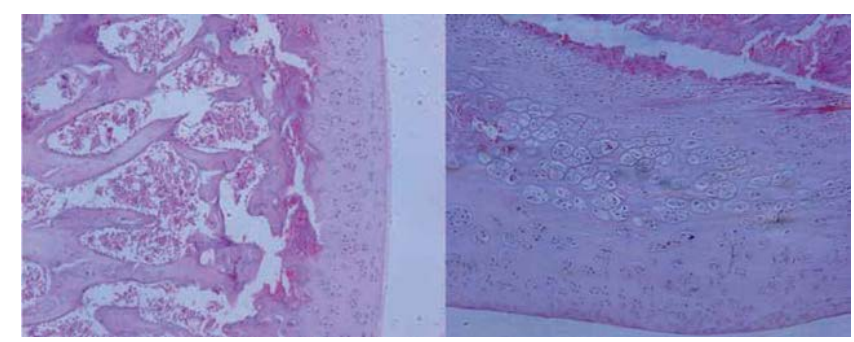

Figure 2: Histological view of the right knee of the male rat after administration of repeated doses of ozone. Natural architecture of the articulation is maintained without signs of inflammation. (From Dr. Emmanouil Iliakis, 2010, personal files).

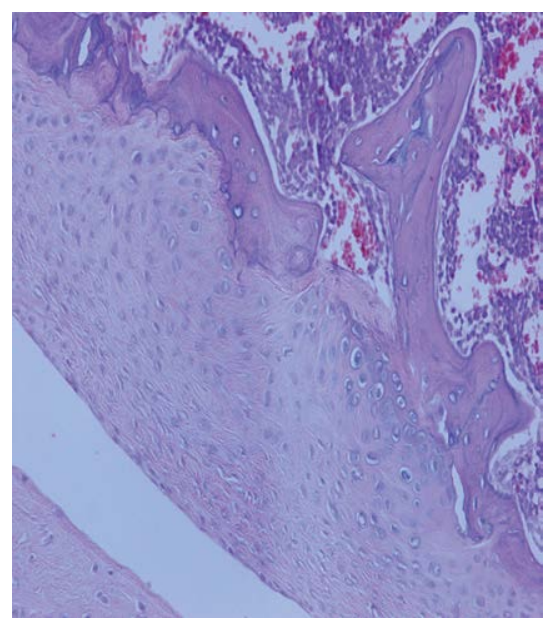

Figure 3: Histology of the articular cartilage of the guinea pig after administration of ozone 7 days after the injection of MIA (group1). Marked reduction of the inflammatory reaction. (From Dr. Emmanouil lliakis, 2011, personal files). 


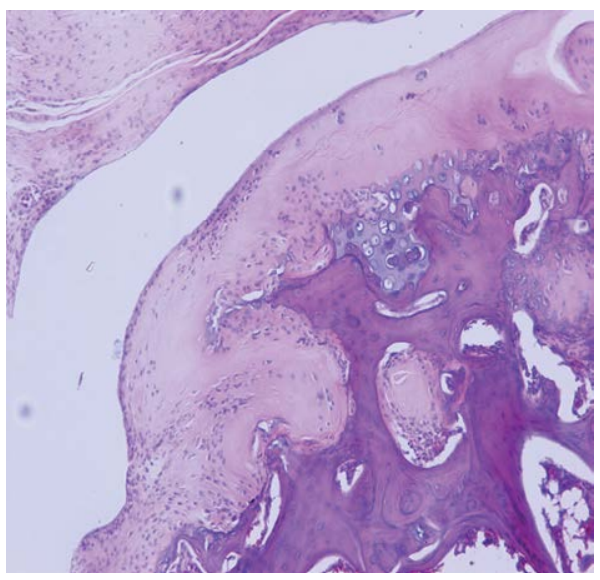

Figure 4: Histology of the knee of the guinea pig after the administration of ozone 14 days after the injection of MIA (group 3). We observe an advanced osteoarthritis with marked signs of inflammation. (From Dr. Emmanouil Iliakis, 2011, personal files)

anti- inflammatory and long term analgesic effects. Intrarticular ozone injections are also effective and costless and a pilot study on the efficacy of the intrarticular ozone therapy for pain control in osteoarthritis of the knee was conducted at the Negev University [38-40].

On the other hand, both large experimental and clinical studies on the effect of ozone therapy are mandatory. Unfortunately our good will is not sufficient to overcome prejudice and lack of sponsors. One relevant possibility is that World Health Authorities, always concerned with the increasing medical costs, could start evaluating a cost-effective ozone therapy and recognize its validity in the integrated treatment of these two relevant pathologies. We believe that the implementation of ozone therapy in all public hospitals, particularly on behalf of patients with little medical assistance, could be a first important step to reduce cost and improve recovery in all chronic oxidative stress diseases.

\section{References}

1. Ray PD, Huang BW, Tsuji Y (2012) Reactive oxygen species (ROS) homeostasis and redox regulation in cellular signaling. Cell Signal 24: 981-990.

2. Halliwell B, Gutteridge JMC (2007) Free Radicals in Biology and Medicine. 4th ed. Oxford University Press; Oxford, UK.

3. Sies H (1985) Oxidative stress: introductory remarks in : H.Sies, Oxidative stress, Academic Press, London, UK.

4. Finkel T, Holbrook NJ (2000) Oxidants, oxidative stress and the biology of ageing. Nature 408: 239-247.

5. Roughley PJ, Alini M, Antoniou J (2002) The role of proteoglycans in aging degeneration and repair of the intervertebral disc. Biochem Soc Trans 30: 869-874.

6. Antoniou J, Steffen T, Nelson F, Winterbottom N, Hollander AP, et al. (1996) The human lumbar intervertebral disc: evidence for changes in the biosynthesis and denaturation of the extracellular matrix with growth, maturation, ageing, and degeneration. J Clin Invest 98: 996-1003.

7. Rutges J, Creemers LB, Dhert W, Milz S, Sakai D, et al. (2010) Variations in gene and protein expression in human nucleus pulposus in comparison with annulus fibrosus and cartilage cells: potential associations with aging and degeneration. Osteoarthritis Cartilage 18: 416-423.

8. Loeser RF (2009) Aging and osteoarthritis: the role of chondrocyte senescence and aging changes in the cartilage matrix. Osteoarthritis Cartilage 17: 971-979.

9. Jallali N, Ridha H, Thrasivoulou C, Butler P, Cowen T (2007) Modulation of intracellular reactive oxygen species level in chondrocytes by IGF-1, FGF, and TGF-beta1. Connect Tissue Res 48: 149-158.

10. Bardia A, Tleyjeh IM, Cerhan JR, Sood AK, Limburg PJ, et al. (2008) Efficacy of antioxidant supplementation in reducing primary cancer incidence and mortality: systematic review and meta-analysis. Mayo Clin Proc 83: 23-34

11. Kamata H, Hirata H (1999) Redox regulation of cellular signalling. Cell Signal 11: 1-14.

12. Rattan SI (2008) Hormesis in aging. Ageing Res Rev 7: 63-78.

13. Bocci V, Borrelli E, Travagli V, Zanardi I (2009) The ozone paradox: ozone is a strong oxidant as well as a medical drug. Med Res Rev 29: 646-682.

14. Borrelli E, Diadori A, Zalaffi A, Bocci V (2012) Effects of major ozonated autohemotherapy in the treatment of dry age related macular degeneration: a randomized controlled clinical study. Int J Ophthalmol 5: 708-713.

15. Borrelli E, Bocci V (2014) Oxygen ozone therapy in the treatment of chronic obstructive pulmonary disease:an integrative approach. American Journal of Clinical and Experimental Medicine 2: 9-13.

16. Borrelli E, De Monte A, Bocci V (2015) Oxygen Ozone therapy in the integrated treatment of chronic ulcer: a case series report. Int J Recent Sci Res 5: 4132 4136

17. Bocci V (2011) Ozone: a new medical drug. $2^{\text {nd }}$ Edition, Springer Verlag, Dordrecht, The Netherlands, Germany.

18. Bocci V, Borrelli E (2015) A practical approach for restoring homeostasis in diseases characterized by a chronic oxidative stress. J Adv Med Pharm Sci 2: 145-153.

19. Bocci VA (2006) Scientific and medical aspects of ozone therapy. State of the art. Arch Med Res 37: 425-435

20. Sagai M, Bocci V (2011) Mechanisms of Action Involved in Ozone Therapy: Is healing induced via a mild oxidative stress? Med Gas Res 1: 29.

21. Pecorelli A, Bocci V, Acquaviva A, Belmonte G, Gardi C, et al. (2013) NRF2 activation is involved in ozonated human serum upregulation of $\mathrm{HO}-1$ in endothelial cells. Toxicol Appl Pharmacol 267: 30-40.

22. Wuertz K, Vo N, Kletsas D, Boos N (2012) Inflammatory and catabolic signalling in intervertebral discs: the roles of NF- $\beta$ and MAP kinases. Eur Cell Mater 23 103-119.

23. Hoyland JA, Le Maitre C, Freemont AJ (2008) Investigation of the role of IL-1 and TNF in matrix degradation in the intervertebral disc. Rheumatology (Oxford) 47: 809-814.

24. Nagano T, Yonenobu K, Miyamoto S, Tohyama M, Ono K (1995) Distribution of the basic fibroblast growth factor and its receptor gene expression in normal and degenerated rat intervertebral discs. Spine (Phila Pa 1976) 20: 1972-1978.

25. Peng B, Hao J, Hou S, Wu W, Jiang D, et al. (2006) Possible pathogenesis of painful intervertebral disc degeneration. Spine (Phila Pa 1976) 31: 560-566.

26. Alexandre A, Buric J, Paradiso R, Salgado H, Murga M et al. (2002) Intradisca injection of $\mathrm{O} 2-\mathrm{O} 3$ to treat hernia disc herniation: results at five years. Riv Ital Ossigeno Ozonoter 1: 165-169.

27. Borrelli E (2011) Mechanism of action of oxygen ozone therapy in the treatment of disc herniation and low back pain. Acta Neurochir Suppl 108: 123-125.

28. Buric J, Rigobello L, Hooper D (2014) Five and ten year follow-up on intradiscal ozone injection for disc herniation. Int J Spine Surg 8.

29. Goldring MB, Goldring SR (2007) Osteoarthritis. J Cell Physiol 213: 626-634.

30. Davies CM, Guilak F, Weinberg JB, Fermor B (2008) Reactive nitrogen and oxygen species in interleukin-1-mediated DNA damage associated with osteoarthritis. Osteoarthritis Cartilage 16: 624-630.

31. DeGroot J, Bank RA, Bijlsma JWJ, TeKoppele JM, Verzij N, et al. (2004) Advanced glycation end products in the development of osteoarthritis. Arthritis ResTher 6: 78-83.

32. Riva Sanseverino E (1989) Knee joint disorders treated by oxygen ozone therapy. Eur Medicophys 25: 163-170.

33. Del Carlo M, Schwartz D, Erickson EA, Loeser RF, et al. (2007) Endogenous production of reactive oxygen species is required for stimulation of human articular chondrocyte matrix metalloproteinase production by fibronectin fragments. Free radic Biol Med 42: 1350-1358

34. Le Maitre CL, Pockert A, Buttle DJ, Freemont AJ, Hoyland JA (2007) Matrix synthesis and degradation in human intervertebral disc degeneration. Biochem Soc Trans 35: 652-655. 
Citation: Borrelli E, Alexandre A, lliakis E, Alexandre A, Bocci V (2015) Disc Herniation and Knee Arthritis as Chronic Oxidative Stress Diseases: The Therapeutic Role of Oxygen Ozone Therapy. J Arthritis 4: 161. doi:10.4172/2167-7921.1000161

Page 6 of 6

35. Peng B, Hao J, Hou S, Wu W, Jiang D, et al. (2006) Possible pathogenesis of painful intervertebral disc degeneration. Spine (Phila Pa 1976) 31: 560-566.

36. Atlas SJ, Keller RB, Wu YA, Deyo RA, Singer DE (2005) Long-term outcomes of surgical and nonsurgical management of sciatica secondary to a lumbar disc herniation: 10 year results from the maine lumbar spine study. Spine (Phila $\mathrm{Pa}$ 1976) 30: 927-935

37. Weinstein AM, Rome BN, Reichmann WM, Collins JE, Burbine SA, et al. (2013) Estimating the burden of total knee replacement in the United States. J Bone Joint Surg Am 95: 385-392.

38. Paoloni M, Di Sante L, Cacchio A, Apuzzo D, Marotta S, et al. (2009)
Intramuscular oxygen -ozone therapy in the treatment of acute low back pain with lumbar disc herniation: a multicenter, randomized, double-blind, clinical trial of active and simulated lumbar paravertebral injection. Spine 34: 13371344

39. Muto M, Andreula C, Leonardi M (2004) Treatment of herniated lumbar disc by intradiscal and intraforaminal oxygen-ozone (O2-O3) injection. J Neuroradiol 31: 183-189.

40. http://clinicaltrials.gov/ct2/show/

NCT00832312?term=ozone+therapyandrank $=2$. 